\title{
The multispecific thyroid hormone transporter OATP1C1 mediates cell-specific sulforhodamine 101-labeling of hippocampal astrocytes
}

\author{
Christian Schnell • Ali Shahmoradi • \\ Sven P. Wichert · Steffen Mayerl • Yohannes Hagos • \\ Heike Heuer • Moritz J. Rossner · Swen Hülsmann
}

Received: 27 July 2013/Accepted: 23 September 2013/Published online: 16 October 2013

(C) The Author(s) 2013. This article is published with open access at Springerlink.com

\begin{abstract}
Sulforhodamine 101 (SR101) is widely used for astrocyte identification, though the labeling mechanism remains unknown and the efficacy of labeling in different brain regions is heterogeneous. By combining region-specific isolation of astrocytes followed by transcriptome analysis, twophoton excitation microscopy, and mouse genetics, we identified the thyroid hormone transporter OATP1C1 as the SR101-uptake transporter in hippocampus and cortex.
\end{abstract}

Keywords Astrocyte - Cell-specific markers ·

Transcriptome analysis

\section{Introduction}

The red fluorescent xanthene derivative sulforhodamine 101 (SR101) has been introduced to neuroscience in 1985 as a dye for activity-dependent labeling of synaptic terminals

Electronic supplementary material The online version of this article (doi:10.1007/s00429-013-0645-0) contains supplementary material, which is available to authorized users.

M. J. Rossner and S. Hülsmann contributed equally to this work.

C. Schnell · A. Shahmoradi - S. P. Wichert .

M. J. Rossner · S. Hülsmann

Center for Nanoscale Microscopy and Molecular Physiology

of the Brain (CNMPB), Göttingen, Germany

C. Schnell · S. Hülsmann $(\square)$

Department of Neurophysiology and Cellular Biophysics,

University Medical Center, Göttingen, Germany

e-mail: shuelsm2@uni-goettingen.de

\section{A. Shahmoradi $\cdot$ S. P. Wichert - M. J. Rossner}

Max-Planck-Institute of Experimental Medicine, Research

Group "Gene Expression and Signaling”, Göttingen, Germany
(Lichtman et al. 1985). Since then, different labeling protocols have been used and thus SR101 was shown to stain different cell types in various regions of the brain with considerable species difference. Reports of labeled cell population range from rabbit oligodendrocytes in the retina (Ehinger et al. 1994) to turtle spinal cord neurons (Mui et al. 2012). In 2004, Nimmerjahn and colleagues identified SR101 as a selective marker for cortical astrocytes in vivo after bolus injection or topical application to the cortical surface (Nimmerjahn et al. 2004). Later, SR101 was also confirmed as a valid method in acutely isolated hippocampal and cortex slices (Ikegaya et al. 2005; Kafitz et al. 2008). However, the molecular mechanism of the cell-specific SR101-labeling remained unknown. Initially, it was hypothesized that gap-junction hemichannels were involved in the labeling process (Nimmerjahn et al. 2004); however, astrocytes from $\mathrm{Cx} 30-/-\mathrm{Cx} 43 \mathrm{fl} / \mathrm{fl}$ :hGFAP-Cre mice (Cx30-/-Cx43-/-, double knockout) that lack most of the gap-junction protein were effectively labeled with SR101 (Pannasch et al. 2011). Recently, pharmacological profiling of SR101 uptake with inhibitors of organic anion transport led to the conclusion that SR101 was likely to be actively transported into astrocytes via an organic anion-transporting

S. Mayerl $\cdot$ H. Heuer

Leibniz Institute for Age Research, Junior Research Group

"Neuroendocrinology", Jena, Germany

Y. Hagos

Department of Systemic Physiology and Pathophysiology,

University Medical Center, Göttingen, Germany

M. J. Rossner $(\square)$

Laboratory of Molecular and Behavioral Neurobiology,

Department of Psychiatry, Ludwig-Maximilians-University

München, Munich, Germany

e-mail: moritz.rossner@med.uni-muenchen.de 
polypeptide (OATP), although the identity of the responsible transporter remained unknown (Schnell et al. 2012).

Furthermore, the use of SR101 has been compromised by recent reports of neuronal SR101-labeling that occurs under pathophysiological conditions such as NMDA receptor activation or ischemia (Thompson et al. 2006; Thompson et al. 2008). Moreover, the side effects of SR101, including chemical LTP, have been reported (Kang et al. 2010; Garaschuk 2013). Therefore, an understanding of the molecular mechanism of SR101 uptake is crucial for its future application as a marker of astrocyte subtypes.

In contrast to the hippocampus, unequivocal identification of astrocytes by SR101 is not possible in the ventral lateral medulla (VLM) of the brainstem, which contains important neurons of the respiratory network. In this region as well as in the rat spinal cord, SR101 has been found to label neurons and astrocytes (Cina and Hochman 2000; Schnell et al. 2012) indicating that a region-specific heterogeneity of astrocytes underlies the lower SR101-uptake efficiencies in the more caudal brain regions.

Based on the pharmacological profile of the SR101labeling (Schnell et al. 2012) we hypothesize that candidate genes from SLC(O) solute carrier families display elevated mRNA expression levels in forebrain versus brainstem astrocytes. To test for this, we captured EGFP-expressing astrocytes from brainstem (poor SR101 uptake) and hippocampus as well as cortex (both efficient SR101 uptake) from hGFAP-EGFP transgenic mice (Nolte et al. 2001) by fluorescence-activated cell sorting (FACS) and performed digital gene expression profiling using next generation sequencing.

\section{Materials and methods}

Breeding of mice

Experiments were performed on acute brain slice preparations of transgenic mice [postnatal day 8-43 (P8-P43), male and female] expressing enhanced green fluorescent protein in astrocytes [TgN (hGFAP-EGFP) GFEC-Fki; (Nolte et al. 2001)] or on Slcolcl knockout mice (Mayerl et al. 2012). Animals were held and bred in the animal facilities of the University Hospital Göttingen and the Leibniz Institute for Age Research in accordance with guidelines of the German Physiological Society as well as the regulations of the State of Lower Saxony and the Federal Republic of Germany.

Slice preparations

Acute transversal slices from brainstem and hippocampus were prepared as described previously (Härtel et al. 2007;
Schnell et al. 2012). In some experiments, parasagittal slices were cut to compare the SR101 staining along the rostral-caudal extension of the cortex. To obtain acute slices, animals were killed by decapitation under deep diethyl-ether anesthesia. The brains were removed from the skull and the isolated hippocampi and brainstem were placed in ice-cooled, carbogen-saturated $\left(95 \% \mathrm{O}_{2}, 5 \%\right.$ $\mathrm{CO}_{2}$ ) artificial cerebrospinal fluid (aCSF) containing $118 \mathrm{mM} \mathrm{NaCl}, 3 \mathrm{mM} \mathrm{KCl}, 1.5 \mathrm{mM} \mathrm{CaCl}_{2}, 1 \mathrm{mM} \mathrm{MgCl}$, $1 \mathrm{mM} \mathrm{NaH}_{2} \mathrm{PO}_{4}, 25 \mathrm{mM} \mathrm{NaHCO} 3$, and $30 \mathrm{mM}$ D-glucose. The osmolarity was $325-335 \mathrm{mosm} / \mathrm{l}$ and the $\mathrm{pH}$ adjusted to 7.4. The isolated brain part was glued with cyanoacryl glue (Loctite Deutschland $\mathrm{GmbH}$ ) to an agar block and mounted in a vibroslicer (VT 1200S, Leica). Slices of 250-400 $\mu \mathrm{m}$ were cut and stored in oxygenated aCSF at room temperature for at least $30 \mathrm{~min}$ before staining. For imaging experiments, slices were transferred to the recording chamber after the staining procedure (see below). Slices were kept submerged by a nylon fiber grid and continuously perfused with aCSF at a flow rate of 5-10 ml/ $\min$.

Sulforhodamine 101 staining protocol

Sulforhodamine 101 (SR101) labeling was performed using the standard protocol as described earlier (Kafitz et al. 2008; Meier et al. 2008; Schnell et al. 2012). Slices were incubated for $20 \mathrm{~min}$ at $34{ }^{\circ} \mathrm{C}$ in carbogenated aCSF containing $1 \mu \mathrm{M}$ SR101 followed by $10 \mathrm{~min}$ in carbogenated aCSF at $34{ }^{\circ} \mathrm{C}$ without SR101 for removal of excess dye from the extracellular space. For the T4/SR101 competition studies, L-Thyroxine (T4; $1-10 \mu \mathrm{M})$ was coapplied with SR101 only during the 20 min incubation period.

Drugs

Electrolytes for aCSF (see above) were purchased from Sigma-Aldrich and Merck chemicals. Drugs were stored in concentrated stock solution at $-20^{\circ} \mathrm{C}$. The $1 \mathrm{mM}$ L-thyroxine sodium salt pentahydrate (Sigma-Aldrich; T2501) stock solution was prepared with $0.1 \mathrm{~N} \mathrm{NaOH}$ and the $0.5 \mathrm{mM}$ SR101 (Sigma-Aldrich, S7635) stock solution was made with distilled water.

Fluorescence imaging using multifocal two-photon excitation microscopy

For detection of EGFP- and SR101 fluorescence, we used a two-photon microscope (TriMScope, LaVision BioTec) with non-descanned detection by GaAsP photomultipliers (Hamamatsu). Two-photon excitation was achieved with a Ti:Sapphire Laser (SpectraPhysics MaiTai BB) at $800 \mathrm{~nm}$. 
Fluorescence signals of hGFAP-EGFP expressing astrocytes were detected through a $531 / 40 \mathrm{~nm}$ band pass emission filter, whereas SR101 fluorescence was detected through a $641 / 75 \mathrm{~nm}$ band pass emission filter (AHF Analysentechnik AG). To allow quantitative comparison of the SR101 intensity between controls and drug treatments, all image parameters, pixel dwell time and number, detector gain as well as laser power were identical for a particular set of experiments. Cell counting was performed in a defined volume that was scanned with $2 \mu \mathrm{m}$ step z-stacks (100 $\mu \mathrm{m}$ in total) using a piezo-focus (Physik Instrumente). All settings were controlled by "Imspector" software (LaVision BioTec).

For quantification of SR101 fluoresence, "Imspector" images were exported to TIFF format. After deconvolution with Autoquant software (MediaCybernetics) using the theoretical point-spread-function (adaptive PSF, ten iterations), further analysis was performed with the Imaris software package (Bitplane) using the "spots" feature of the "surpass" view mode. In this mode, astrocytes were identified by their EGFP fluorescence in the green channel and a spherical 3D volume (spot) of $6 \mathrm{~mm}$ diameter was assigned to the soma of each EGFP-positive cell astrocytes.

To quantify the SR101-labeling, the green channel was turned off. The "recentering" function was used to correct the position of the spot in the red SR101 channel. If this was impossible or if the SR101 intensity was not differing from the surrounding background signal, the particular cell was counted as an SR101-negative cell. The parameter "SR101positive astrocytes (\%)" was calculated by dividing the number of both SR101-positive and EGFP-positive cells by the total number of EGFP-positive cells multiplied by 100 . SR101- and EGFP-fluorescence intensities of individual cells were derived from the median intensity of the assigned spot and averaged for each slice individually. The parameter "SR101 intensity (\% of EGFP fluorescence)" was calculated by dividing the SR101 intensity by the EGFPfluorescence intensity multiplied by 100 .

Analysis of imaging data

Fluorescence intensity and cell number are presented as means and standard error (SEM). One-way ANOVA tests with multiple comparisons versus control group (Bonferroni $t$ test) were used to determine statistical significance, considering $P \leq 0.05$ as significant. Statistical calculations were performed in SigmaPlot (Systat Software GmbH).

Fluorescent activated cell sorting (FACS) of EGFPpositive astrocytes

Brains were removed from P10 old hGFAP-EGFP mice (Nolte et al. 2001) and sectioned in oxygenated aCSF at
$4{ }^{\circ} \mathrm{C}$ with a vibratome to obtain $150 \mu \mathrm{m}$ thick slices. Cortex, hippocampus and brainstem were subsequently dissected and processed by incubation with freshly prepared Papain solution for $20 \mathrm{~min}$ at $37{ }^{\circ} \mathrm{C}$ as suggested by the manufacturer (Worthington). Samples were homogenized by gentle trituration with a $1 \mathrm{ml}$ glass pipette, followed by staining of nuclei with cell-permeable nuclear Hoechst 33,342 dye (Sigma) at $37{ }^{\circ} \mathrm{C}$ for $10 \mathrm{~min}$. Cells were filtered through $40 \mu \mathrm{m}$ nylon cell strainer before sorting. FACS isolation of EGFP-positive astrocytes was done using a FACS Aria II (Becton-Dickinson) and gates were set according to control samples for each brain region from wild-type mice. First, forward and side scatter was used to deplete for small sized cellular debris, followed by selection of Hoechst 33,342 and EGFP double-positive cells which were directly sorted into RLT lysis buffer (Qiagen) and frozen at $-20{ }^{\circ} \mathrm{C}$ until use.

Amplification of RNA and generation of libraries and Illumina sequencing

For generation of libraries for Illumina sequencing from pools of sorted EGFP-positive astrocytes, we modified an established linear amplification protocol using the DNAdependent RNA polymerase from bacteriophage T7 (Rossner et al. 2006). Therefore, we first isolated RNA using RNA easy microcolumns (Qiagen) according to manufacturer protocols except of RNA elution, which was done by adding $100 \mu \mathrm{H}_{2} \mathrm{O}$ followed by ethanol precipitation with $\mathrm{NH}_{4} \mathrm{Ac}$ (f.c. $2.5 \mathrm{M}$ ) and pellet paint (Invivogen) as carrier. The precipitate was air-dried and resolved in $2 \mu \mathrm{l}$ of freshly diluted oligo-dT T7-B primer $(2 \mu \mathrm{M})$. cDNA was synthesized in a total volume of $4 \mu \mathrm{l}$ using a $2 \times$ pre-mix of Superscript III polymerase $\left(1 \mathrm{~h} 50{ }^{\circ} \mathrm{C}\right)$ according to manufacturer's recommendations (Invitrogen). Double-stranded (ds) cDNA was initiated by adding RNAseH and DNA PolI in a volume of $26 \mu$ las described (Rossner et al. 2006). Purification of ds cDNA with GFX columns (GE Healthcare) and T7-mediated antisense RNA amplification (T7 Megascript Kit, Ambion) and aRNA purification were essentially performed as described (Rossner et al. 2006). Second-round cDNA synthesis was initiated with $2 \mu \mathrm{l}$ of a random hexamer primer $(100 \mu \mathrm{M})$ with hairpins flanking a sequence stretch for PCR amplification (Dec1-Hairpin-N9), which was shown to suppress false-positive PCR products (Adli et al. 2010) in a volume of $10 \mu \mathrm{l}$ with Superscript III for $10 \mathrm{~min}$ at $25^{\circ} \mathrm{C}$ followed by $50 \mathrm{~min}$ at $50{ }^{\circ} \mathrm{C}$. RNA was subsequently degraded by adding $\mathrm{NaOH}\left(25 \mathrm{~min}\right.$ at $\left.68^{\circ} \mathrm{C}\right)$ and neutralized by $\mathrm{HCl}$ (1 $\mu$ l of a $25 \mathrm{mM}$ solution each). Advantage Taq polymerase (Clontech) and primer B-short were used to generate ds cDNA in a volume of $100 \mu$ l (according to the manufacturer's recommendations) and purified using 
Nucleo-Spin columns (Macherey \& Nagel). Pool-identifying barcodes were introduced by PCR using PWO polymerase (Roche) and Code_Dec1 c40 and B-short as primers for ten cycles in a volume of $20 \mu \mathrm{l}$ and an annealing temperature of $58{ }^{\circ} \mathrm{C}$. PCR products were analyzed by agarose gel electrophoresis and combined to a final sample for the attachment of Illumina sequencing adaptors by five cycles of PCR with PWO (Roche) and $1 \mu \mathrm{l}$ of each primer (Illumina-for and Illumina-B-rev at $10 \mu \mathrm{M}$ ) in a volume of $100 \mu \mathrm{l}$. Size selection (200-400 bp) of the final PCR product was done by agarose gel electrophoresis and gel extraction using the Nucleospin extraction kit (Macherey \& Nagel). Sequencing of libraries was performed at the Genome Center Cologne of the Max-PlanckSociety according to established protocols on an HiSEQ2000 machine (Illumina) at a depth of 150 Mio reads in total. For primer sequences and graphical representation of the protocol, see supplementary table.

Processing of reads and bioinformatic analysis

FastQ files were converted into fastA files with the FASTQ-to-FASTA converter and split into pools according to barcodes using the FASTQ/A Barcode splitter (both tools were from http://hannonlab.cshl.edu/fastx_toolkit/), trimmed to 35 bases and mapped to the mouse using the Mus musculus NCBI Built 37.1. Differential expression analysis was performed using the Bioconductor package DESeq (Version 1.4.1) (Anders and Huber 2010).

\section{Results}

Weak astroglial SR101-labeling in the brainstem

In an initial set of experiments, we compared SR101 staining from different regions of the brainstem, midbrain and hippocampus using [TgN (hGFAP-EGFP)] mice at ages between P8 and P23. The SR101 intensity in all the tested regions of the brainstem was weak, especially when compared with the labeling in the hippocampus (Fig. 1). There was some regional heterogeneity also within the brainstem, with more intense labeling in the lateral superior olive (LSO; Fig. 1k), but altogether the SR101-labeling of EGFP-positive astrocytes was far below the level in hippocampal EGFP-positive cells. Additionally, we tested SR101-labeling along the rostral-caudal extension of the cortex of sagittal slices (Fig. 2). The astroglial SR101labeling at the three tested cortical areas was indistinguishable from each other. Thus, this test proved that our approach of sorting EGFP-positive astrocytes from hippocampus (Hi) (and cortex; $\mathrm{Cx}$ ) and brainstem will be valid to detect a putative transporter for SR101.
Differential expression of Oatplc1 in astrocytes from forebrain over brainstem

Based on the hypothesis that the putative SR101 transporter belongs to the solute carrier (organic anion transporter) family (Schnell et al. 2012), we focused the analysis of our next-generation sequencing results on these genes (slc or slco gene symbols). Comparing the mRNA levels of astrocytes from hippocampus (Hi), cortex $(\mathrm{Cx})$ and brainstem (BS), we identified 359 transporter mRNAs (mapped refSeq IDs, see methods) coding for 276 proteins that were expressed at least in one of the three different regions $(\geq 10$ reads, see supplementary table). However, we detected only seven mRNAs encoding for five transporters of solute carrier families including the glial glutamate transporter 1 (Glt1/Slcla2), the sodium calcium exchanger ( $N c x 2 /$ Slc8a2) and the vesicular glutamate transporter 1 (Vglut1/ Slc17a7) that were expressed higher in the forebrain $(\mathrm{Cx}$ and $\mathrm{Hi}$ ) vs. brainstem astrocytes (Fig. 3d). There was no organic anion transporter (OAT; Slc22 family) among these genes, but from the Slco family, we found mRNAs for two isoforms of the organic anion transporting polypeptide (Oatp)1c1 (Slcolcl) that were differentially expressed between forebrain and brainstem astrocytes $(>2$ fold increase, adjusted $p$ value $<0.05$ ). Additionally there were ten genes that showed higher mRNA levels in the brainstem as compared to the $\mathrm{Cx}$ and $\mathrm{Hi}$, including the known astroglial transporters for inhibitory neurotransmitter, GAT3 (encoded by Slc6all) and GlyT1 (encoded by Slc6a9; supplementary table.)

Thyroid hormone inhibition of SR101-labeling in hippocampal astrocytes

Oatplc1/Slcolcl has been identified to code for a thyroid hormone transporter(OATP14, OATP-F; Pizzagalli et al. 2002) and, indeed, this candidate was the only one with a very similar pharmacological profile (Sugiyama et al. 2003) as described for the putative SR101 uptake system in hippocampal slices (Schnell et al. 2012). To test if the physiological substrate of OATP1C1, L-thyroxine (T4), can compete with the SR101 uptake, we incubated acute hippocampal slices (P21-P22) in SR101 in the presence of different concentrations of T4 $(1-10 \mu \mathrm{M} ; n=4)$. The intensity of SR101 fluorescence in EGFP-labeled astrocytes of the stratum radiatum was significantly reduced in a dose-dependent manner (Fig. 4a-e). The relative number of EGFP-positive cells, which showed some detectable, although very low level of SR101 fluorescence (SR101-positive cells), however, did not follow this dose dependency (Fig. 4f). Taken together, the observed reduction in intensity of SR101-labeling supported our hypothesis that OATP1C1 is the transporter responsible for SR101 uptake into astrocytes. 
Fig. 1 SR101 uptake in various regions of the forebrain and hindbrain. a-c Coronal diagrams of the brain section analyzed; $\mathbf{d}$ schematic drawing showing rostro-caudal position of diagrams a-c (panels ad were adapted and modified from the Paxinos Atlas of the developing mouse brain (Paxinos 2007). e-p Figures are maximal intensity projections of 3D 2-photon scans $(100 \mu \mathrm{m} z$ stack, 51 sections) taken from mice at P8. $\mathbf{e}^{\prime}-\mathbf{p}^{\prime}$ The corresponding EGFP expression is shown below the SR101 image. $C A 1$ corpus ammonis of hippocampus, $A P T$ anterior pretectal nucleus, $P o$ posterior thalamic nuclear group, $\mathrm{PnC}$ pontine reticular nucleus, caudal part, $5 \mathrm{~N}$ motor trigeminal nucleus, $L S O$ lateral superior olive, $\operatorname{Pr} 5 V L$ principal sensory trigeminal nucleus, ventrolateral part, $p y$ pyramidal tract, $p B C$ pre-Bötzinger complex, $12 \mathrm{~N}$ hypoglossal nucleus, $I O$ inferior olivary nucleus. Scale bars $40 \mu \mathrm{m}$
A
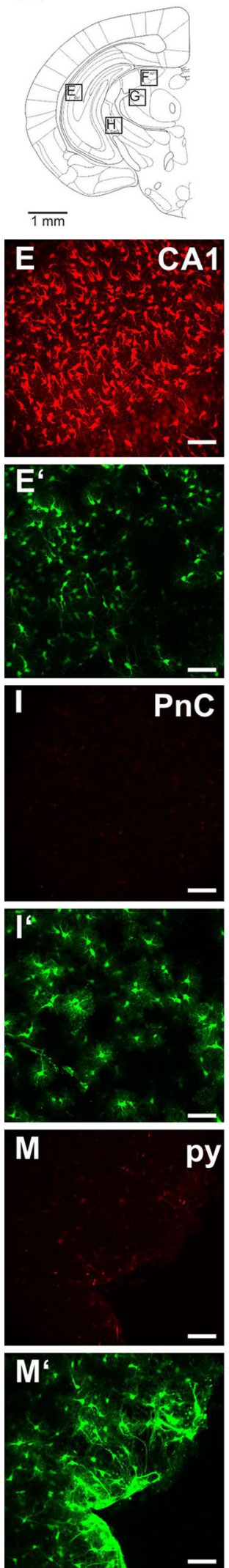

B
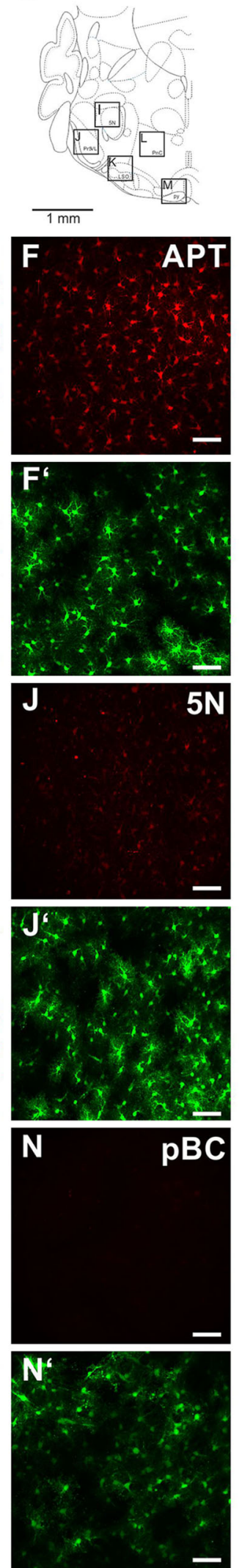

C

D
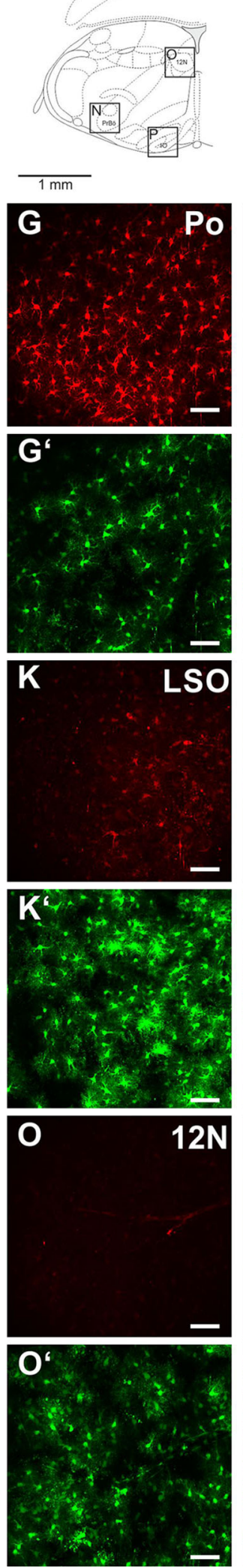
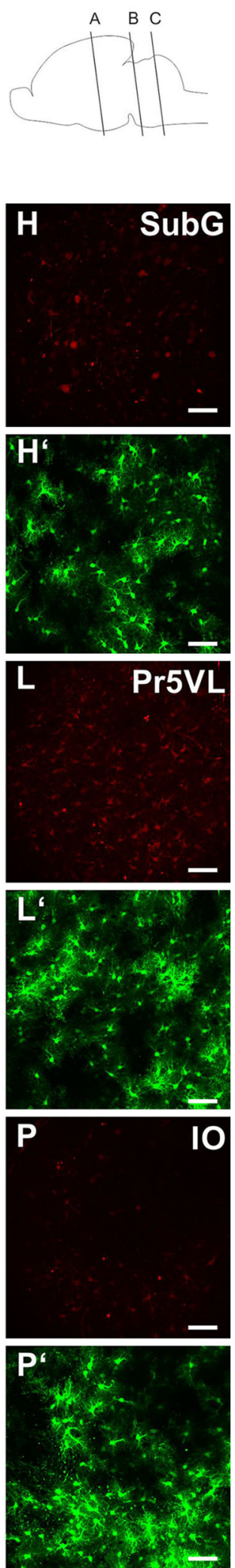

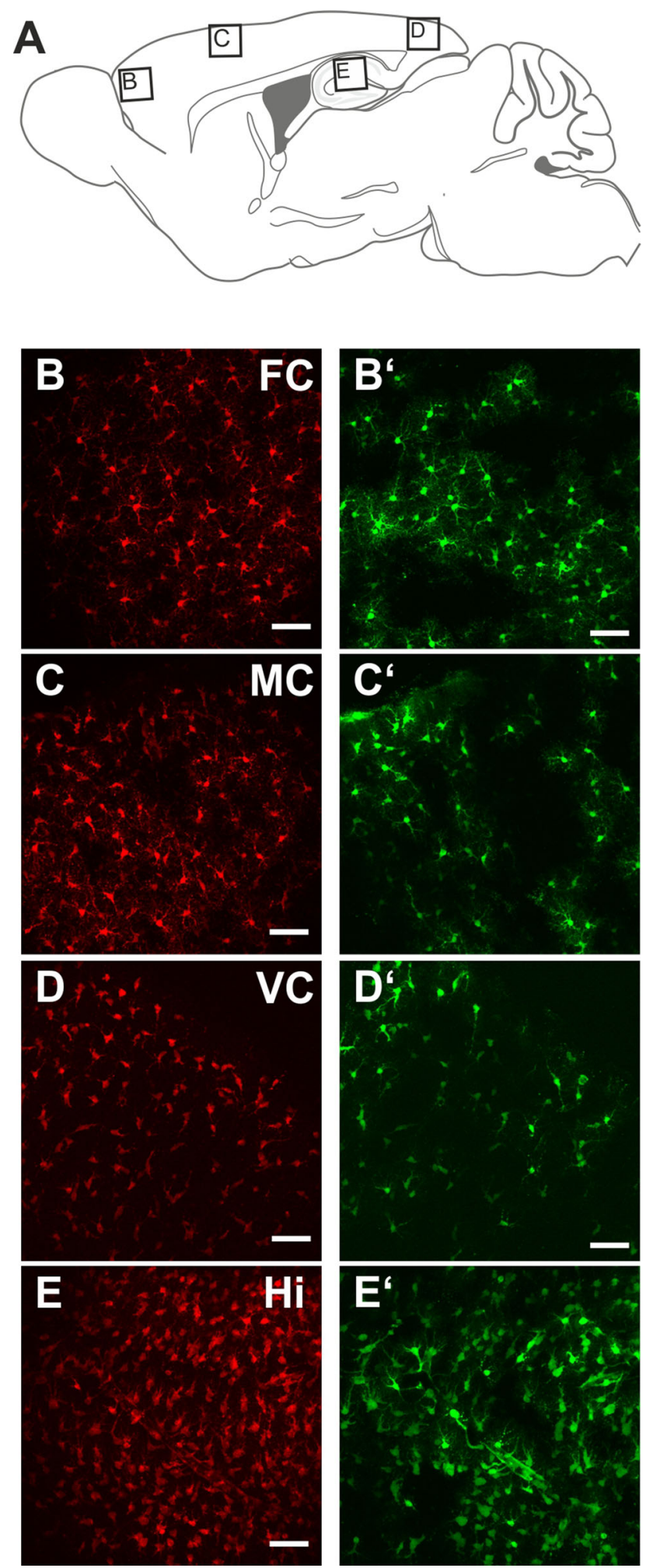

Lack of SR101-labeling of astrocytes from Oatp1c1 knockout mice

To further substantiate this assumption that OATP1C1 is the astroglial SR101-uptake transporter, we analyzed
4Fig. 2 Comparison of SR101 uptake along the rostral-caudal extension of the cortex. a Schematic drawing showing rostro-caudal position of images $\mathbf{b}-\mathbf{e}$ (panel $\mathbf{a}$ was adapted and modified from the Paxinos Atlas (Paxinos et al. 2001). b-e SR101-labeling as maximal intensity projections of the 3D two-photon scan $(100 \mu \mathrm{m} z$-stack, 51 sections) from $\mathbf{b}$ the frontal associative cortex $(F C)$, $\mathbf{c}$ motor cortex $(M C)$ and $\mathbf{d}$ visual cortex $(V C)$. Scans were taken from parasagittal slices (P10, 1-2 mm lateral to the midline). Additionally, a maximal intensity projection image from the hippocampus $(\mathrm{Hi})$ is depicted in (e). $\mathbf{b}^{\prime}-\mathbf{e}^{\prime}$ The corresponding EGFP expression is shown next to the SR101 image. Scale bars $40 \mu \mathrm{m}$

SR101 uptake in Oatplc1 null mutant (Slcolc1-/-) mice (Mayerl et al. 2012). In hippocampal slices from Slcolc1-/- mice, the identification of astrocytes by SR101 is impossible (Fig. 4g-h). Since we could not identify any SR101-labeled cell that morphologically resembled an astrocyte, we further concluded that the weak labeling observed in astrocytes of the ventrolateral medulla (Schnell et al. 2012) could in fact result from the low, albeit robustly detected level of expression of Oatp14/ Slcolcl in the brainstem compared to hippocampus and cortex (Fig. 3d). In this context, it is interesting to note that the unspecific labeling that we sometimes observed in the superficial neurons of the CA1 pyramidal layer (Fig. 4 and supplementary movie) can still be observed after T4 application (Fig. 4b) and in the knockout slices (Fig. 4h).

\section{Discussion}

SR101 uptake in hippocampal astrocytes is mediated by $O A T P 1 C 1$

Oatplc1/Slcolc1 cDNA, coding for OATP1C1 that is also known as OATP-F and OATP14, was cloned in 2008 as part of the blood-brain barrier genome program (Chu et al. 2008). This high-affinity transporter for thyroxine (T4) acts sodium-independently in a bidirectional manner (Pizzagalli et al. 2002; Sugiyama et al. 2003; Tohyama et al. 2004). Apart from T4, other OATP1C1 substrates, like probenecid, estrone-3-sulfate (Tohyama et al. 2004) or dehydroepiandrosterone (Sugiyama et al. 2003) reduced SR101 uptake in hippocampal slices (Schnell et al. 2012). Although OATP1C1 expression on the endothelial side of the bloodbrain barrier is established, the function of OATP1C1 as a putative astrocytic transporter for T4 uptake is still under debate (Grijota-Martínez et al. 2011; Ridder et al. 2011; Heuer and Visser 2013). Our experiments finally prove the expression of OATP1C1 in subpopulations of astrocytes. Moreover, the overlap of OATP1C1 expression with Aquaporin 4 (Roberts et al. 2008) suggests a preferential targeting of the transporter to astroglial end feet of the blood brain barrier. This polarized expression of OATP1C1 expression on end feet together with the endothelial 

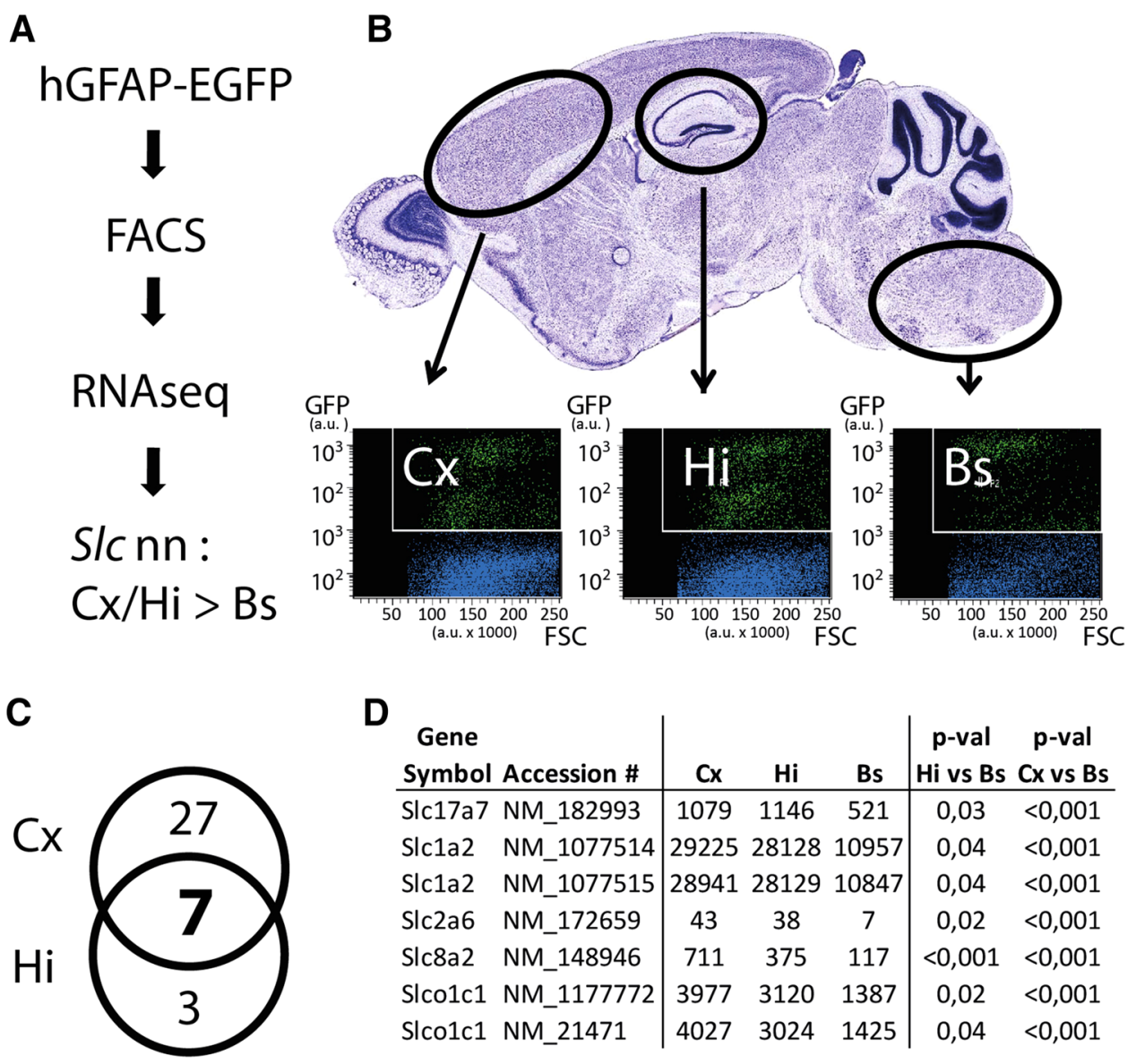

\begin{tabular}{|c|c|c|c|c|c|c|}
\hline $\begin{array}{l}\text { Gene } \\
\text { Symbol }\end{array}$ & Accession \# & $C x$ & $\mathrm{Hi}$ & Bs & $\begin{array}{c}\text { p-val } \\
\text { Hi vs Bs }\end{array}$ & $\begin{array}{c}\text { p-val } \\
\text { Cx vs Bs }\end{array}$ \\
\hline Slc17a7 & NM_182993 & 1079 & 1146 & 521 & 0,03 & $<0,001$ \\
\hline Slc1a2 & NM_1077514 & 29225 & 28128 & 10957 & 0,04 & $<0,001$ \\
\hline Slc1a2 & NM_1077515 & 28941 & 28129 & 10847 & 0,04 & $<0,001$ \\
\hline Slc2a6 & NM_172659 & 43 & 38 & 7 & 0,02 & $<0,001$ \\
\hline Slc8a2 & NM_148946 & 711 & 375 & 117 & $<0,001$ & $<0,001$ \\
\hline Slcolc1 & NM_1177772 & 3977 & 3120 & 1387 & 0,02 & $<0,001$ \\
\hline Slcolc1 & NM_21471 & 4027 & 3024 & 1425 & 0,04 & $<0,001$ \\
\hline
\end{tabular}

Fig. 3 Identification of transporter genes enriched in forebrain astrocytes by transcriptome analysis. a Experimental strategy: cortex $(\mathrm{Cx})$, hippocampus $(\mathrm{Hi})$ and brainstem $(\mathrm{Bs})$ tissues from brains of mice expressing EGFP under the control of the glial-specific promoter GFAP $(h G F A P-E G F P)$ were triturated and $50 \mathrm{k}$ EGFP-positive cells were purified by FACS, followed by RNA isolation and transcriptome profiling with RNAseq. SR101 candidate uptake transporter genes (of the solute carrier, Slc family) were hypothesized to display a higher expression in $\mathrm{Cx}$ and $\mathrm{Hi}$ versus Bs astrocytes $(\mathrm{Cx} / \mathrm{Hi}>\mathrm{Bs})$. b Scatter plots of FACS analysis with GFP intensities plotted versus forward scatter (FSC) given as arbitrary units (a.u.). Blue dots represent Hoechst 33,342 positive and GFP negative cells, green dots represent

expression (Fig. 5) explains why SR101 can cross the blood brain barrier and label astrocytes when injected into the vasculature (Appaix et al. 2012). The absence of astroglial SR101-labeling in the Oatp1c1-/- mice suggests that exclusively OATP1C1 mediates astrocytic SR101 uptake in hippocampal astrocytes.

A general, region-specific heterogeneity of astrocytes

Our next-generation sequencing (NGS) approach not only allows us to identify the uptake transporter for SR01 that explains the region-specific differences of SR101-labeling observed in the cardio-respiratory regions of the brainstem (Schnell et al. 2012), but also sheds some light on a more
Hoechst 33,342 and GFP double-positive cells representing different astrocyte populations. c Venn diagram of all forebrain enriched transporter genes. $27(\mathrm{Cx})$ and $3(\mathrm{Hi})$ refseq annotated mRNAs were detected to be significantly higher expressed in $\mathrm{Cx}$ or $\mathrm{Hi}$ astrocytes ( $>2$ fold change), and seven mRNAs were significantly elevated both in $\mathrm{Cx}$ and $\mathrm{Hi}$. d Differentially expressed mRNAs coding for solute carriers (Slc and Slco gene families). The gene symbols, accession and average read numbers in $\mathrm{Cx}, \mathrm{Hi}$ and Bs samples are depicted. The seven detected forebrain enriched mRNAs correspond to five genes (Slcla2 and Slcolcl annotated by two mRNAs each). Detection cutoff was determined with an adjusted $p$ value cutoff $<0.05$. Cx $(n=2)$, Hi $(n=3)$, Bs $(n=4)$ biological replicates

general heterogeneity of astrocytes. We could detect differences in the expression of neurotransmitter transporters that have already been reported before as for the glycine transporter 1 (Slc6a9), GAT3 (Slc6a11) or the glial glutamate transporter GLT1 (Slcla2), a finding that underscores the reliability of our method (Supplementary table). Since expression of the transporter apparently correlates with the importance of the different transmitter systems in these brain regions, it is tempting to speculate that differential expression of the OATP1C1 across the forebrain and brainstem is based on a more active involvement of forebrain astrocytes in the control of local thyroid hormone metabolism.

Interestingly, in parallel to the forebrain-enriched expression of the astroglial thyroid hormone transporter 


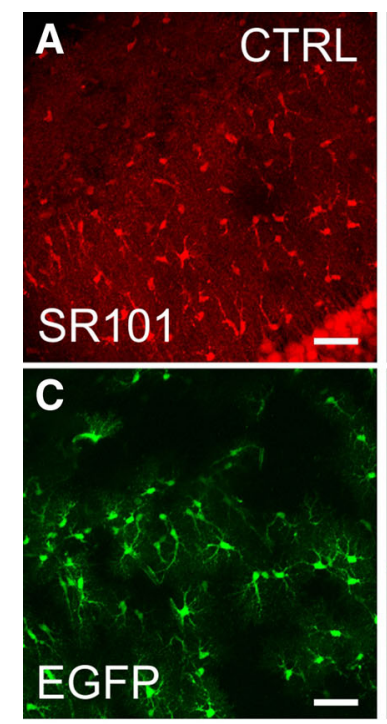

E

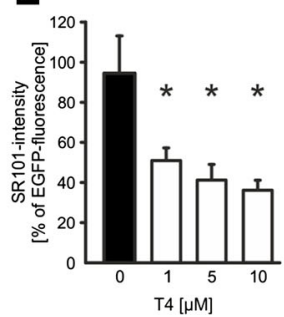

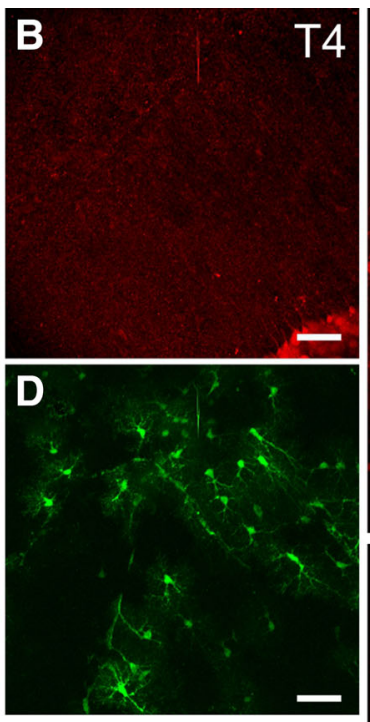

$\mathbf{F}$

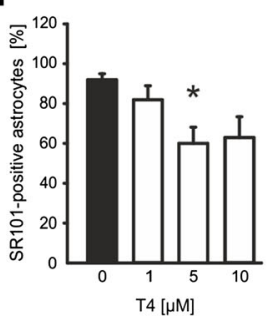

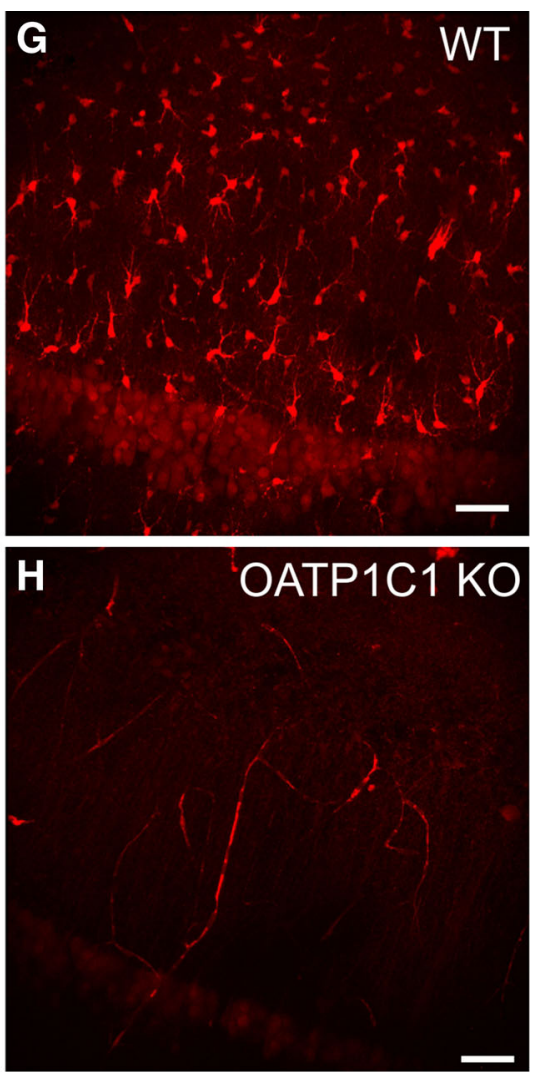

Fig. 4 The thyroid hormone transporter OATP1C1 (SLCO1C1) is the astrocytic uptake transporter for SR101. a-d Maximal intensity projections of SR101-labeling hippocampus slices from hGFAPEFGP mouse (P22) under control conditions (CTRL a) and in the presence of $10 \mu \mathrm{M}$ levothyroxine during the 20 min loading period (T4 b). Corresponding EGFP fluorescence is shown in green below the SR101 figure (c, d). e, $\mathbf{f}$ The basic statistical description of the blockade of SR101 uptake by T4 is shown in (e) for the SR101 intensity as relative to the EGFP fluorescence. $\mathbf{f}$ Comparison of the fraction of EGFP-positive astrocytes with a SR101-fluorescence signal larger than background (SR101-positive astrocytes). Data are

Oatp1c1, we also found a higher expression of the type 2 iodothyronine deiodinase (Dio2), the enzyme that converts thyroxine (T4) to 3,3',5-triiodothyronine (T3), in the cortex and the hippocampus as compared to Bs (Cx 14,564, Hi 8,120 , Bs 3,947 norm. reads; adjusted $p$ values $\mathrm{Cx}$ vs. Bs $<0.001$ and $\mathrm{Hi}$ vs. $\mathrm{Bs}=0.0886)$. Further investigation will be necessary to unveil the relevance of astroglial function and eventually brain development, but the differential expression of OATP1C1 appears to be a rather general difference that is even preserved in humans. Indeed, human OATP1C1 shows a region-specific expression with preferential detection of mRNA in the forebrain over the hindbrain (Pizzagalli et al. 2002).

\section{Methodological considerations}

As in a preceding paper (Schnell et al. 2012), we observed differences in the significance levels when comparing the given as mean $\pm \operatorname{SEM}$ ( $n=4$ slices, four animals; one way ANOVA with multiple comparisons versus Control Group; Bonferroni $t$ test). Significance is indicated by an asterisk for $p<0.05$. g, h SR101 uptake is diminished in Oatplcl knockout mice. While astrocytes were labeled by SR101 in Hi slices from WT mice from the same strain (three animals, $n=5$ slices), no SR101-labeling was observed in hippocampus slices from Slco1c1 KO mice (five animals; $n=15$ slices). Note that the unspecific labeling in the superficial neurons of the CA1 pyramidal layer (Thompson et al. 2006) is still present after $\mathrm{T} 4$ and in the knockout slices

reduction of SR101 intensity, which was significant for all T4 concentrations tested, with the apparent numbers of SR101-labeled EGFP-positive astrocytes. Due to the manual detection (see methods), which we found to be superior to the automatic detection, all astrocytes with SR101 intensities that are just above the background are counted and every EGFP-positive astrocyte with an incomplete blockade of the transporter counts as an SR101-positive cell. Therefore, we have to assume that the SR101 uptake by the OATP1C1 is not completely blocked by the applied concentrations of T4. However, in the Oatplc1-/- slice, we were unable to detect individual cell somata in the SR101 channel, suggesting that there was no alternative route of SR101 uptake in hippocampal astrocytes.

Our sequencing experiments were performed on FACSisolated EGFP-expressing cells from TgN (hGFAP-EGFP) mice. The majority of the EGFP-expressing cells have been proven to be astrocytes in the brain regions from which we 


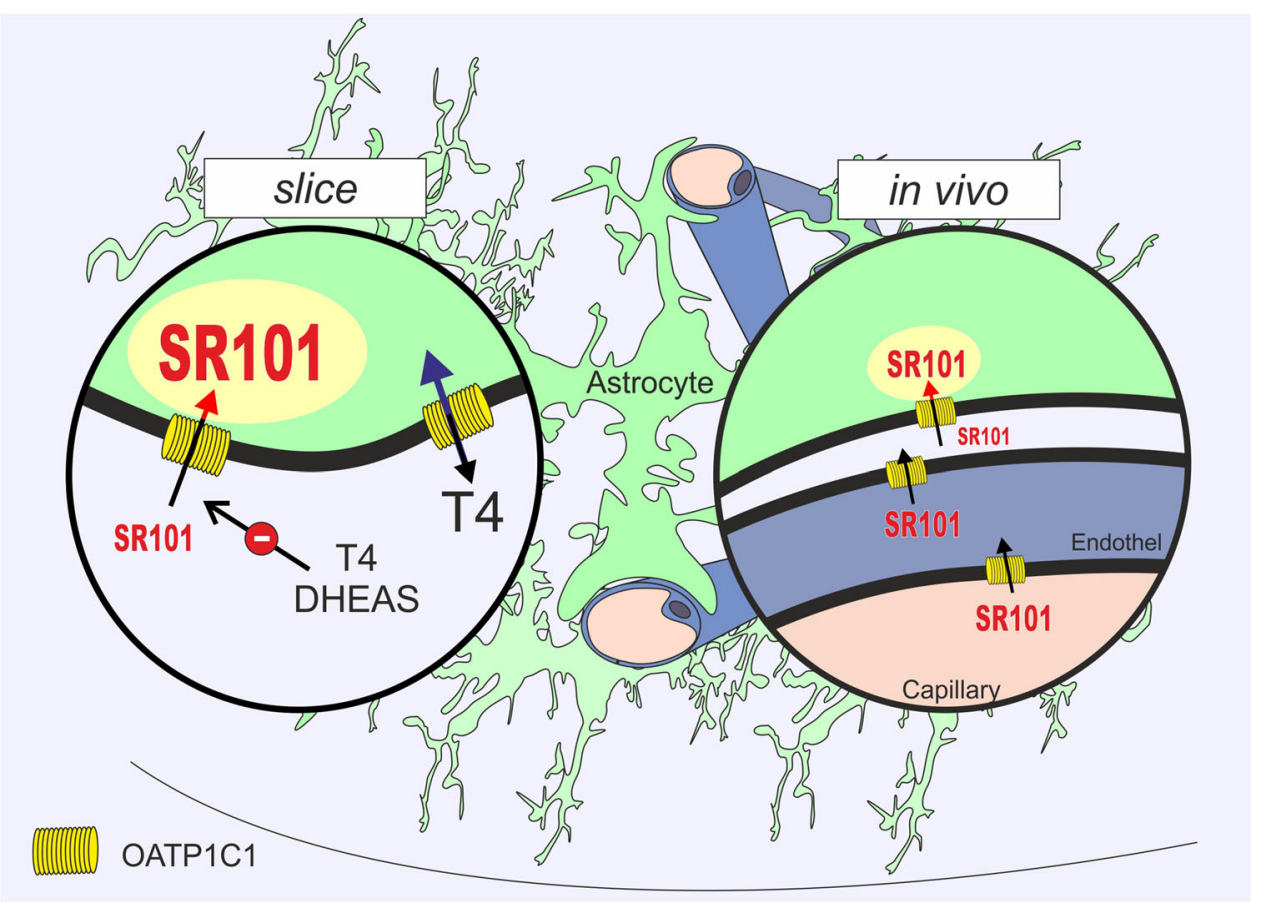

Fig. 5 Schematic of the SR101-labeling mechanism. The figure summarizes the mechanism of SR101-labeling in astrocytes. In slices (left) SR101 is taken up into astrocytes that express the thyroid hormone transporter OATP1C1 (Yellow). Uptake is blocked by levothyroxine (T4; see Fig. 4a-f) and neurosteroids e.g., dehydroepiandrosterone sulfate [DHEAS; (Schnell et al. 2012)]. Furthermore (right in vivo), Oatp1c1 is also expressed on the vascular endothelium

took FACS samples (Nolte et al. 2001; Grass et al. 2004). When interpreting the mRNA data, however, one must be aware that the FACS-sorted EGFP-positive cells from the hippocampus might include some neural precursors, e.g., from the subgranular zone of the dentate gyrus, which are also EGFP positive (Hüttmann et al. 2003). Additionally, the EGFP-labeled cells population also includes a subpopulation of NG2-positive cells (oligodendrocyte precursor cells) that also were shown to be EGFP-positive in the hippocampus (Matthias et al. 2003; Jabs et al. 2005) as well as in the brainstem (Grass et al. 2004; Szöke et al. 2006). For our aim, the identification of the SR101 transporter, however, contamination with non-astrocyte cell populations was not critical, since we confirmed these results with pharmacological experiments and by using the Slcolcl knockout mouse.

\section{Unspecific neuronal labeling in the CA1-pyramidal layer}

In some of our experiments, we noticed unspecific labeling of pyramidal neurons close to the slice surface (see Fig. 3 and supplementary movie). Such unspecific neuronal labeling has been reported before, although using different labeling conditions, including lower temperature
(Ridder et al. 2011) allowing uptake of SR101 from the blood stream (Appaix et al. 2012). Since SR101 still labels vessels in the Oatp1c1 knockout mouse (Fig. 4h), there might be a second transporter that transports SR101 into the endothelium. Alternatively, there might be also unspecific binding of SR101 to vasculature, as described, e.g., for Alexa Fluor 633 (Shen et al. 2012)

(Nimmerjahn and Helmchen 2012) or reduced extracellular magnesium concentration (Thompson et al. 2008) or hypoxia (Thompson et al. 2006). From the observation that unspecific staining was also found after application of $\mathrm{T} 4$ or in the OATP1C-deficient situation (Fig. 4), one might conclude that the unspecific neuronal staining results from another mechanism that is not available or active in the astrocyte. To answer the question whether this involves the opening of pannexin hemichannels, as suggested (Thompson et al. 2006, 2008), further experiments are requires that are beyond the scope of the paper.

\section{Perspectives}

Although our study primarily aimed at identifying the uptake mechanism of SR101 into astrocytes that underlies the regional difference of SR101-labeling, we would like to emphasize that by matching pharmacological properties with cell type-specific expression profiles of transporters, other dyes or compounds could be rationally designed and/ or identified to selectively target additional cell types of the brain. Moreover, our strategy can pave the way toward a rational design of cell type-selective dyes that may be used for basic research purposes and also in the context of diagnostic and therapeutic approaches for, e.g., gliomas, 
which often have special expression profiles for OATPS (Bronger et al. 2005).

\section{Conclusion}

We have demonstrated that hippocampal astrocytes take up sulforhodamine 101 via the thyroid hormone transporter OATP1C1. This observation will help to understand the possible side effects of SR101 application and prevent pitfalls when using the drug in novel preparations or under modified condition.

Acknowledgments We acknowledge the excellent technical support by Elena Ciirdaeva (Göttingen, Germany) for NGS library generation. The authors are also grateful to Anja-Annett Grützner for excellent technical assistance. This work was supported by the "Deutsche Forschungsgemeinschaft" (DFG grants HU797/5-1 and KFO241-RO4076/1-1 and the DFG-Research Center for Nanoscale Microscopy and Molecular Physiology of the Brain (CNMPB)). The funders had no role in study design, data collection and analysis, decision to publish or preparation of the manuscript.

Conflict of interest The authors declare a potentially competing financial interest: $\mathrm{YH}$ is Chief Executive Officer (CEO) of PortaCellTec Biosciences $\mathrm{GmbH}$, a company that provides hepatic/renal uptake transporter assays. This does not alter the authors' adherence to all the journals policies on sharing data and materials.

Open Access This article is distributed under the terms of the Creative Commons Attribution License which permits any use, distribution, and reproduction in any medium, provided the original author(s) and the source are credited.

\section{References}

Adli M, Zhu J, Bernstein BE (2010) Genome-wide chromatin maps derived from limited numbers of hematopoietic progenitors. Nat Meth 7:615-618

Anders S, Huber W (2010) Differential expression analysis for sequence count data. Genome Biol 11:R106

Appaix F, Girod S, Boisseau S, Römer J, Vial J-C, Albrieux M, Maurin M, Depaulis A, Guillemain I, van der Sanden B (2012) Specific in vivo staining of astrocytes in the whole brain after intravenous injection of sulforhodamine dyes. PLoS ONE 7:e35169

Bronger H, König J, Kopplow K, Steiner H-H, Ahmadi R, HeroldMende C, Keppler D, Nies AT (2005) ABCC drug efflux pumps and organic anion uptake transporters in human gliomas and the blood-tumor barrier. Cancer Res 65:11419-11428

Chu C, Li JY, Boado RJ, Pardridge WM (2008) Blood-brain barrier genomics and cloning of a novel organic anion transporter. J Cereb Blood Flow Metab 28:291-301

Cina C, Hochman S (2000) Diffuse distribution of sulforhodaminelabeled neurons during serotonin-evoked locomotion in the neonatal rat thoracolumbar spinal cord. J Comp Neurol 423:590-602

Ehinger B, Zucker CL, Bruun A, Adolph A (1994) In vivo staining of oligodendroglia in the rabbit retina. Glia 10:40-48
Garaschuk O (2013) Imaging microcircuit function in healthy and diseased brain. Exp Neurol 242:41-49

Grass D, Pawlowski PG, Hirrlinger J, Papadopoulos N, Richter DW, Kirchhoff F, Hulsmann S (2004) Diversity of functional astroglial properties in the respiratory network. J Neurosci 24:1358-1365

Grijota-Martínez C, Díez D, Morreale de Escobar G, Bernal J, Morte B (2011) Lack of action of exogenously administered T3 on the fetal rat brain despite expression of the monocarboxylate transporter 8. Endocrinology 152:1713-1721

Härtel K, Singaravelu K, Kaiser M, Neusch C, Hülsmann S, Deitmer JW (2007) Calcium influx mediated by the inwardly rectifying $\mathrm{K}+$ channel Kir4.1 (KCNJ10) at low external $\mathrm{K}+$ concentration. Cell Calcium 42:271-280

Heuer H, Visser TJ (2013) The pathophysiological consequences of thyroid hormone transporter deficiencies: insights from mouse models. Biochim Biophys Acta 1830:3974-3978

Hüttmann K, Sadgrove M, Wallraff A, Hinterkeuser S, Kirchhoff F, Steinhauser C, Gray WP (2003) Seizures preferentially stimulate proliferation of radial glia-like astrocytes in the adult dentate gyrus: functional and immunocytochemical analysis. Eur $\mathbf{J}$ Neurosci 18:2769-2778

Ikegaya Y, Le Bon-Jego M, Yuste R (2005) Large-scale imaging of cortical network activity with calcium indicators. Neurosci Res $52: 132-138$

Jabs R, Pivneva T, Huttmann K, Wyczynski A, Nolte C, Kettenmann H, Steinhauser C (2005) Synaptic transmission onto hippocampal glial cells with hGFAP promoter activity. J Cell Sci 118:3791-3803

Kafitz KW, Meier SD, Stephan J, Rose CR (2008) Developmental profile and properties of sulforhodamine 101-Labeled glial cells in acute brain slices of rat hippocampus. J Neurosci Methods 169:84-92

Kang J, Kang N, Yu Y, Zhang J, Petersen N, Tian GF, Nedergaard M (2010) Sulforhodamine 101 induces long-term potentiation of intrinsic excitability and synaptic efficacy in hippocampal CA1 pyramidal neurons. Neuroscience 169:1601-1609

Lichtman JW, Wilkinson RS, Rich MM (1985) Multiple innervation of tonic endplates revealed by activity-dependent uptake of fluorescent probes. Nature 314:357-359

Matthias K, Kirchhoff F, Seifert G, Huttmann K, Matyash M, Kettenmann H, Steinhauser C (2003) Segregated expression of AMPA-type glutamate receptors and glutamate transporters defines distinct astrocyte populations in the mouse hippocampus. J Neurosci 23:1750-1758

Mayerl S, Visser TJ, Darras VM, Horn S, Heuer H (2012) Impact of Oatp1c1 deficiency on thyroid hormone metabolism and action in the mouse brain. Endocrinology 153:1528-1537

Meier SD, Kafitz KW, Rose CR (2008) Developmental profile and mechanisms of GABA-induced calcium signaling in hippocampal astrocytes. Glia 56:1127-1137

Mui JW, Willis KL, Hao ZZ, Berkowitz A (2012) Distributions of active spinal cord neurons during swimming and scratching motor patterns. J Comp Physiol A Neuroethol Sens Neural Behav Physiol 198:877-889

Nimmerjahn A, Helmchen F (2012) In vivo labeling of cortical astrocytes with sulforhodamine 101 (SR101). Cold Spring Harbor protocols 2012:326-334

Nimmerjahn A, Kirchhoff F, Kerr JN, Helmchen F (2004) Sulforhodamine 101 as a specific marker of astroglia in the neocortex in vivo. Nat Meth 1:31-37

Nolte C, Matyash M, Pivneva T, Schipke CG, Ohlemeyer C, Hanisch UK, Kirchhoff F, Kettenmann H (2001) GFAP promotercontrolled EGFP-expressing transgenic mice: a tool to visualize astrocytes and astrogliosis in living brain tissue. Glia 33:72-86 
Pannasch U, Vargová L, Reingruber J, Ezan P, Holcman D, Giaume C, Syková E, Rouach N (2011) Astroglial networks scale synaptic activity and plasticity. Proc Natl Acad Sci 108:8467-8472

Paxinos G (2007) Atlas of the developing mouse brain: at E17.5, PO, and P6. Elsevier, Amsterdam u.a.

Paxinos G, Franklin KBJ, Franklin KBJ (2001) The mouse brain in stereotaxic coordinates. Academic Press, San Diego

Pizzagalli F, Hagenbuch B, Stieger B, Klenk U, Folkers G, Meier PJ (2002) Identification of a novel human organic anion transporting polypeptide as a high affinity thyroxine transporter. Mol Endocrinol 16:2283-2296

Ridder DA, Lang M-F, Salinin S, Röderer J-P, Struss M, Maser-Gluth C, Schwaninger M (2011) TAK1 in brain endothelial cells mediates fever and lethargy. J Exp Med 208:2615-2623

Roberts LM, Woodford K, Zhou M, Black DS, Haggerty JE, Tate EH, Grindstaff KK, Mengesha W, Raman C, Zerangue N (2008) Expression of the thyroid hormone transporters monocarboxylate transporter-8 (SLC16A2) and organic ion transporter-14 (SLCO1C1) at the blood-brain barrier. Endocrinology 149:6251-6261

Rossner MJ, Hirrlinger J, Wichert SP, Boehm C, Newrzella D, Hiemisch H, Eisenhardt G, Stuenkel C, von Ahsen O, Nave KA (2006) Global transcriptome analysis of genetically identified neurons in the adult cortex. J Neurosci 26:9956-9966
Schnell C, Hagos Y, Hulsmann S (2012) Active sulforhodamine 101 uptake into hippocampal astrocytes. PLoS ONE 7:e49398

Shen Z, Lu Z, Chhatbar PY, O'Herron P, Kara P (2012) An arteryspecific fluorescent dye for studying neurovascular coupling. Nat Meth 9:273-276

Sugiyama D, Kusuhara H, Taniguchi H, Ishikawa S, Nozaki Y, Aburatani H, Sugiyama Y (2003) Functional characterization of rat brain-specific organic anion transporter (Oatp14) at the blood-brain barrier: high affinity transporter for thyroxine. J Biol Chem 278:43489-43495

Szöke K, Härtel K, Grass D, Hirrlinger PG, Hirrlinger J, Hülsmann S (2006) Glycine transporter 1 expression in the ventral respiratory group is restricted to protoplasmic astrocytes. Brain Res 1119:182-189

Thompson RJ, Zhou N, MacVicar BA (2006) Ischemia opens neuronal gap junction hemichannels. Science 312:924-927

Thompson RJ, Jackson MF, Olah ME, Rungta RL, Hines DJ, Beazely MA, MacDonald JF, MacVicar BA (2008) Activation of pannexin-1 hemichannels augments aberrant bursting in the hippocampus. Science 322:1555-1559

Tohyama K, Kusuhara H, Sugiyama Y (2004) Involvement of multispecific organic anion transporter, Oatp14 (Slc21a14), in the transport of thyroxine across the blood-brain barrier. Endocrinology 145:4384-4391 